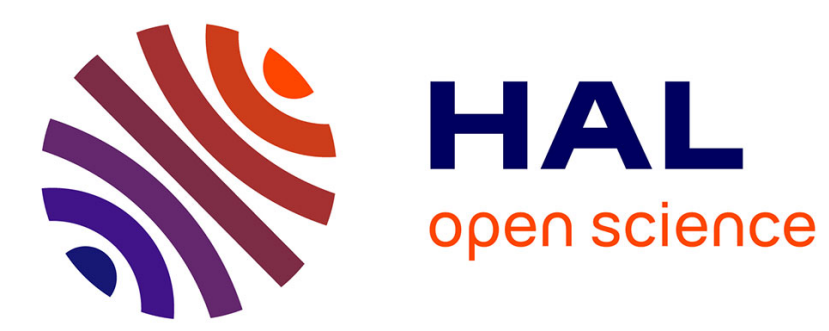

\title{
INTERNAL FRICTION AND ACOUSTIC EMISSION DUE TO CHARGE DENSITY WAVES
}

\author{
M. Doyama
}

\section{To cite this version:}

M. Doyama. INTERNAL FRICTION AND ACOUSTIC EMISSION DUE TO CHARGE DENSITY WAVES. Journal de Physique Colloques, 1985, 46 (C10), pp.C10-669-C10-676. 10.1051/jphyscol:198510148 . jpa-00225354

\section{HAL Id: jpa-00225354 https://hal.science/jpa-00225354}

Submitted on 1 Jan 1985

HAL is a multi-disciplinary open access archive for the deposit and dissemination of scientific research documents, whether they are published or not. The documents may come from teaching and research institutions in France or abroad, or from public or private research centers.
L'archive ouverte pluridisciplinaire HAL, est destinée au dépôt et à la diffusion de documents scientifiques de niveau recherche, publiés ou non, émanant des établissements d'enseignement et de recherche français ou étrangers, des laboratoires publics ou privés. 
JOURNAL DE PHYSTQUE

Colloque C10, supplément au $\mathrm{n}^{\circ} 12$, Tome 46, décembre 1985 page C10-669

\title{
INTERNAL FRICTION AND ACOUSTIC EMISSION DUE TO CHARGE DENSITY WAVES
}

\author{
M. DOYAMA
}

Department of Metallurgy and Materials Science, Faculty of Engineering, The University of Tokyo, 7-3-1 Hongo, Bunkyo-ku, Tokyo 113, Japan

\begin{abstract}
Much attention has been paid to the charge density waves which are observed in low-densional conductors. The elastic behavior in connection with the formation of charge density waves is reviewed. The change in internal friction due to the formation of charge density wawes is discussed. Results on a few experiments on the acoustic emission due to the formation of charge density waves are presented.
\end{abstract}

\section{1- INTRODUCTION}

Low dimensional materials have received considerable attention in recent years because of their characteristic properties based on the low dimensionality. The natural materials which have layered structures such as mica or graphite have been known for many years.

Recently some materials are artificially synthesized, for example TTF. TCNQ (Tetrathiafulvalence-Tetracyanoquinodimethane) as one dimensional structure polymers, one dimensional structure transition metal trichalcogenides, two dimensional structure transition metal dichalcogenides and graphite intercalation. Some of the great interests are concerned with the Peierls transition, charge density waves and super conductors based on the Frohlich mechanism. Consider a one-dimensional metal conductor. Let the length of a unit cell be $a$. The relation between the kinetic energy of conduction electrons $E$ and the wave number $\mathrm{k}$ is given by $\mathrm{Fig} . \mathrm{l}(\mathrm{a})$. When the lattice is distorted with a period of $D$ which is larger than a, then energy gap can be created as shown in Fig. 1 (b).

If the conduction electron system is stabilized by this lattice distortion, and the stabilized energy is larger than the energy of lattice distortion, then this process spontaneously occurs. The net energy gain may be the largest when the period of lattice distrotion $D$ satisfies the condition $k_{F}= \pm \mathrm{mm} / \mathrm{D}$, where $k_{F}$ is the Fermi wave number, $m$ is an integer. If this energy gap is form-

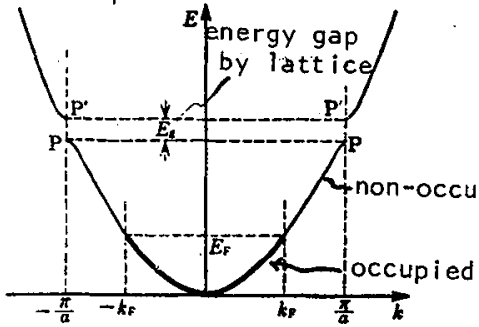

(a)

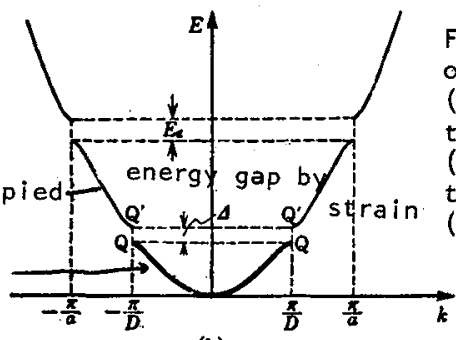

(b)
Fig. 1. Energy gap in a one-dimensional lattice (a) With periodic potential (lattice parameter a (b) With periodic potential and periodic strain ( period D). 
ed at the Fermi energy, the conductor becomes an insulator. This is the Peierls transition. At higher temperatures, this energy gap will disappear because of the disturbance due to the thermal energy of lattice vibrations. The relation has been given, where $\quad k T=1.14 E_{B} \exp (-1 / \lambda)$

$k$ is the Boltzmann constant, $E_{B}$ is about the energy of the band width, $v_{0}$ the perturbation potential, $w_{0}$ frequency of the lattice, $N$ the total number of unit cells, $D\left(E_{F}\right)$ the density of states at Fermi energy. The band gap $E(T)$ is written as $\Delta E(T)=\left(\Delta^{2} D\left(E_{F}\right) / 2\right) \ln \left(1.14 E_{B} /(k T)\right)$

for one dimensional metals. $\triangle$ is a constant representing a matrix element. Figure 2 (a) shows the lattice distortion, Figs. 2 (b) and (c) show the stable and unstable electron distribution probabilities, which correspond to $Q$ and $Q^{\prime}$ states in Fig. l(b), respectively. This electron wave of conduction and rarefaction is called charge density wave (CDW). When the wave length of charge density wave becomes a simple multiple of lattice periodicity, the CDW is called commensurate CDW (CCDW), when it is not a simple multiple, the CDW is called inommensurate CDW (ICDW). The elastic strain is lower for the commensurate CDW than ICDW. Therefore ICDW of ten changes to CCDW at a lower temperature. The transition from ICDW to CCDW is called the lock-in of CDW. The speed of electrons at Fermi level is usually quite fast, much faster than the velocity of lattice waves. The group velocity of electrons, however, is zero. The electron system is stabilized by the lattice distortion of special wave length. For the lattice vibration of this wave length, the distortion becomes easier. The elastic constant becomes lower or the lattice becomes softer for this $v i$ bration of this wave length. This effect is distinct, particularly for one dimensional conductors as shown in Fig. 3. This is called giant Kohn anomaly.

The elastic constant changes at the CDW transition temperature. This effect has been measured in many low dimensional materials. In some materials, pretransformation changes, even above the transition temperature have been observed, such as the changes in the lattice constant or lattice softening.

At absolute zero Keivin, CDW has a definite amplitude and phase. At a finite temperature the amplitude and phase of CDW have fluctuations. These are called amplitude mode and phase mode, respectively. The phase mode is called a phason. The dispersion relation of the modes $(q-w)$ are shown in Fig. 4. The order parameter of CDW can be written as $\psi=A \exp (i \phi)$ where $A$ is the amplitude and $\phi$ is the phase. Above T'(CDW transition temperature) $\psi=0$.

To satisfy eq (4), there are two possibilities. 1) the amplitude A becomes zero or 2) the phase $\phi$ becomes random.

Transition metals ( $M$ : $\mathrm{Ti}, \mathrm{Zr}, \mathrm{Hf} ; \mathrm{V}, \mathrm{Nb}, \mathrm{Ta} ; \mathrm{Mo}, \mathrm{W}$ ) and chalcogens form $M \times 2$ and $M \times 3$ compounds. MX2's are the layered dichalcogenides which extibit twodimensional anisotropic properties and associated electronic instabilities. Electron-diffraction studies revealed the existence of super lattice structures which are attributed to the formation of charge-density waves. The metal coordination within a layer may be octahedral or trigonal prismatic, and the

(a)

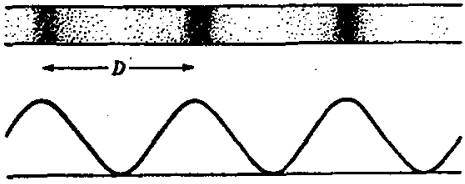

(c)

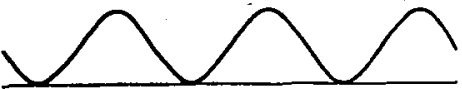

Fig.2. Two standing wave:states caused by periodic lattice strains (a) Lattice longitudinal waves, (b) Electron distribution of stable standing waves, (c) Electron distribution of unstable standing waves.

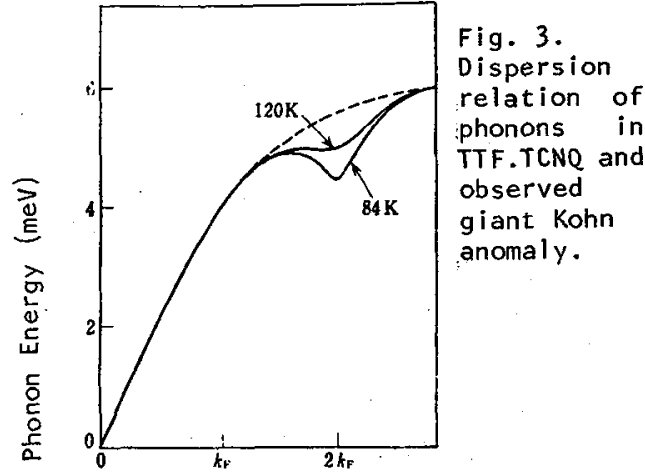

Wave number of phonons $q$ 
layers are stacked in various ways, polytypes $(1 \mathrm{H}, 2 \mathrm{H}, 4 \mathrm{Hb}, \ldots . .$.$) . The basic$ structure of layered transition metal dichalcogenides is that the hexagonal transition metal lattice is sandwiched by two layers of hexagonal chalcogens. This basic structure is multiplyed by stacking. There are two basic structures, octahedral and trigonal prism types (Fig. 5). IT type consists of octahedral basic structures, only. $2 \mathrm{H}$ and $3 \mathrm{R}$ trigonal prisms only. In $4 \mathrm{H}(\mathrm{b}) 6 \mathrm{R}$, octahedral and trigonal prism are mixed. Transition tri-chalcogenids form whiskers and exhibit one-dimensional anisotropic properties. The electrical conductivity is much higher in the direction of the axis of whiskers but much lower in the perpendicular directions to the axis of whiskers. The crystal structure of CDW phases have been studied by electron diffraction and electron microscopy. The interrelation of the elastic modulus, thermal expansion coefficients, and.specific heat has been calculated $/ 1 /$.

Sato et al /2/ measured the momentum distribution of electrons in IT-TaS2 and $2 \mathrm{H}-\mathrm{TaSe} 2$ for the two crystal directions as a function of temperature by the Doppler broadening technique of positron annihilation. In IT-TaS2, an observed change at $220 \mathrm{~K}$ is ascribed to the lattice expansion along the c-axis, and the other change at $350 \mathrm{~K}$ to the vanishing of a part of the Fermi surface, both are caused by the commensuration of CDW (Fig. 6). Below 100K an effect of Anderson localization was observed. In $2 \mathrm{H}-\mathrm{TaSe} 2$ a clear change was observed below $122 \mathrm{~K}$ which is ascribed to the onset of CDW (Fig. 7).

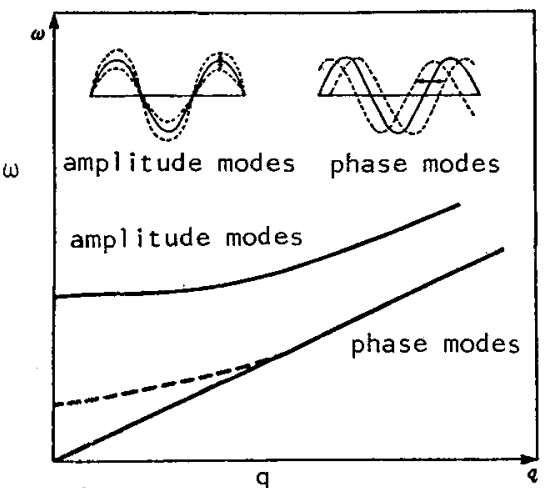

Fig. 4. Collective modes of CDW. (a)

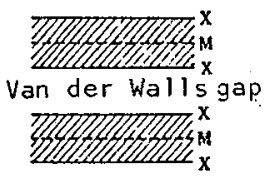

(b)
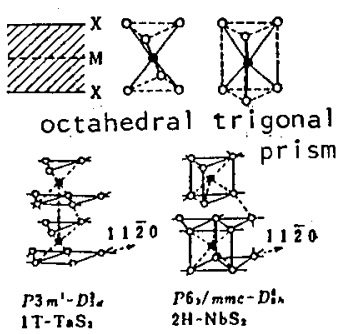

Fig. 5 .

Crystal structure of $M X_{2}$

(a) băsic type

(b) polytype

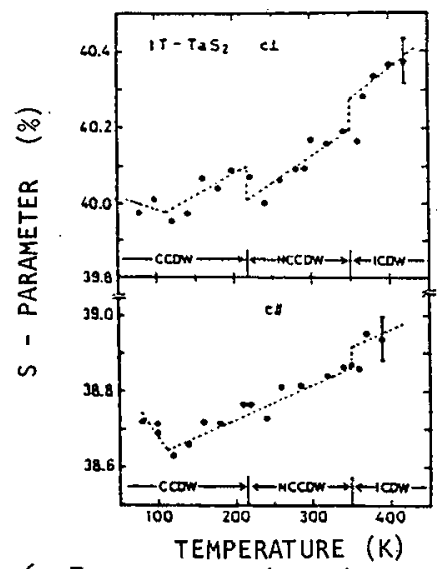

Fig.6. Temperature dependence of the Doppler broadening $S$-parameter $/ 2 /$.

Fig.7. Temperature dependence of the Doppler broadening $S$-parameter along the conducting direction (c); upper curve, and along the perpendicular direction $(c / /)$; lower curve, in $2 \mathrm{H}-\mathrm{TaSe}_{2} / 2 /$. 


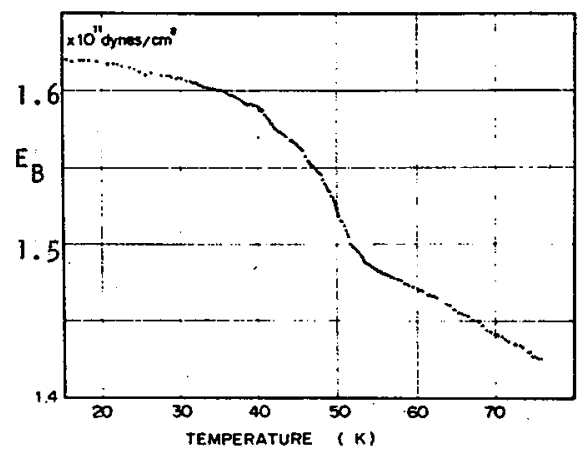

Fig.8. Temperature dependence of Young's modulus along the b-axis. TTF. TCNQ /4/.

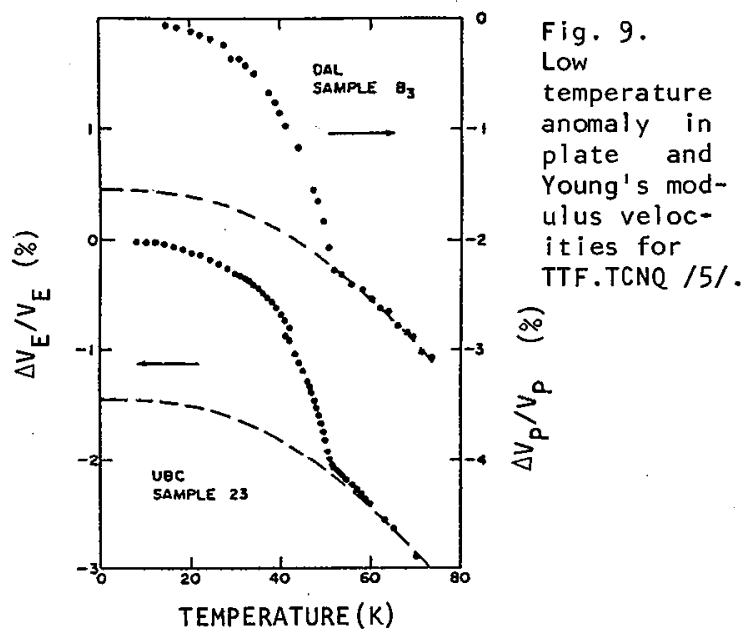

11 - CHANGES IN ELASTIC CONSTANTS DUE TO CDW TRANSITIONS

The elastic constants change at the CDW transition temperatures.

TTF-TCNQ: Barmatz et al /3/ observed an anomaly of Young's modulus in TTF-TCNQ at about $35 \mathrm{~K}$. I shiguro et al $/ 4 /$ measured the change in Young's modulus along the b-axis in TTF-TCNQ as shown in Fig. 8. They observed an anomaly at 53.5K corresponding to the anomaly in the electrical conductivity. This anomaly is related to the metal-non metal transition. The sound velocities as a function of temperature have been measured as shown in Fig. $9 / 5 /$.

2H-TaSe2: The temperature dependence of the modulus in $2 \mathrm{H}-\mathrm{TaSe} 2$ is shown in Figs.10,11,12 /6/. A small elastic anomaly occurs at the incommensurate transition near $12 \mathrm{lk}$, while a much larger anomaly takes place at the commensurate transition near 90K. A small hysteresis has been found at $121 \mathrm{~K}$ transition,this indicates that this transition is a second order transition. Unusual feature of the modulus behavior at the ICDW transition in TaSe2 is the shape of its temperature dependence. The temperature dependence is expected to be the same as the specific heat anomaly near the transition temperature. Figure 12 shows a large elastic hysteresis $(\approx 5 K)$ which clearly demonstrates the first-order character of $2 \mathrm{H}-\mathrm{TaSe} 2$ 90K transition. Figure 12 shows that the differnece in the temperature of the modulus minimum at each frequency indicates that elastic hysteresis at this transition is frequency dependent. Elastic anomalies are also observed at $122 \mathrm{~K}$ (TO) and $90 \mathrm{~K}$ (Td) $/ 7 /$.

2H-NbSe2:Barmatz et al/6/ measured the change in the elastic constant in NbSe2. Figure 13 shows the temperature dependence of the reed modulus in $2 \mathrm{H}-\mathrm{NbSe} 2$.

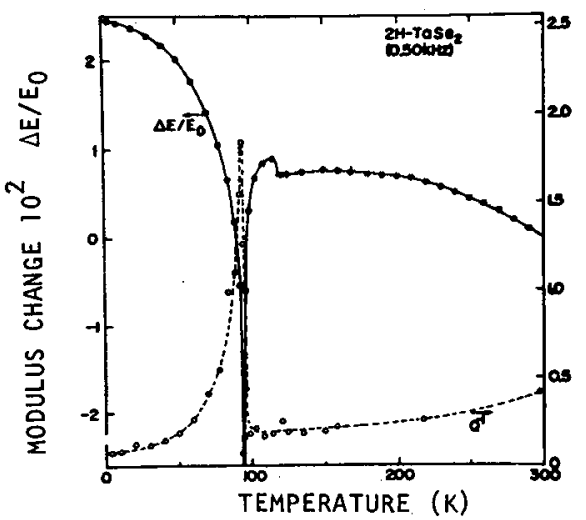

Fig.10. Temperature dependence of the modulus and internal friction in $2 \mathrm{H}-$ $\mathrm{TaSe}_{2} / 6 /$.

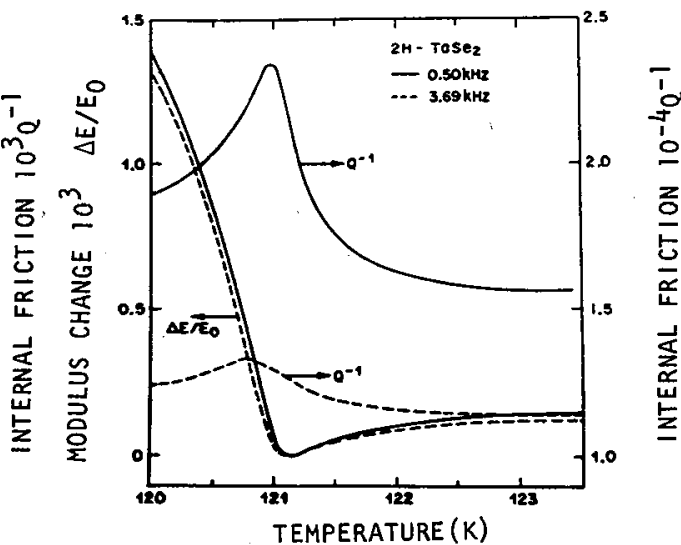

Fig.11. Modulus and internal friction near the incommensurate transition in $2 \mathrm{H}-\mathrm{TaSe}_{2} / 6 /$. 


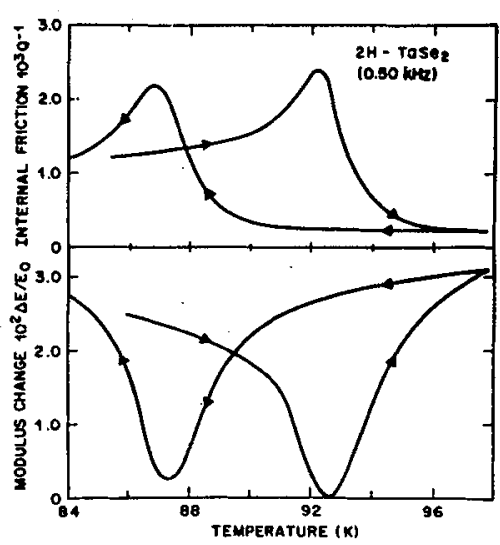

Fig.12. Continuous heating and cooling curves of the elastic properties near the commensurate transition in $2 \mathrm{H}-\mathrm{TaSe}_{2} / 6 /$.

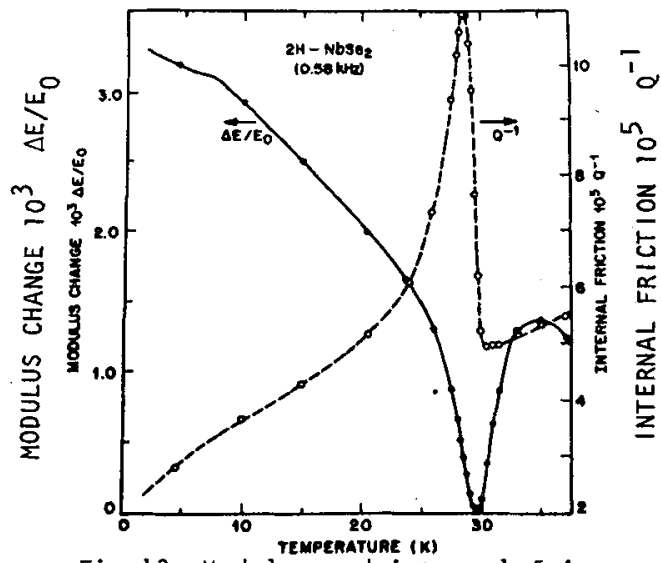

Fig.13. Modulus and internal friction near the incommensurate transition in $2 \mathrm{H}-\mathrm{NbSe}_{2} / 6 /$.

The modulus reaches a minimum at the incommensurate CDW transition at $29.8 \mathrm{~K}$. The hysteresis was fairly large, about $5 K$; this indicates that this transition is the first order. Skolnick et al/8/ measured the apparent velocity of transverse sound propagating perpendicularly to the layer planes in NbSe2. They found an anomaly at the region around the incommensurate CDW phase transition. TaS3: Brill /9/ measured the change in the modulus in TaSe 3 near the charge density wave transition at $222 \mathrm{~K}$. The modulus has a large dip ( $2 \%$ ) at the transition. The transition was the second order unlike NbSe3.0rthorhombic TaS 3 (o-TaS3) undergoes a charge density wave transition at 220K. A number of unusual transport properties, most prominently non-Ohmic conductivity $/ 10 \%$, when the electric field in the sample exceedsa threshold field ET, which is sample and temperature dependent. A variety of physical models had success in describing these phonomena in terms of CDW motion. The CDW is pinned to the lattice defects for fields E ET and becomes depinned for E ET. Brill and Roark $/ 11 /$ measured the modulus under electric field. They found the decrease in the modulus. This suggests the depinning of CDW.

NbSe3: Brill and Ong $/ 12 /$ measured Young's modulus of NbSe3. An anomaly in Young's modulus was observed at $T 1$ (142K) but not at $T 2$ (58K). These transitions are second order because no hysteresis was observed. At a second-order phase transition, the change in Young's modulus Ei (measured along the ith direction) is related to the specific heat anomaly, $\Delta c p$

$\left(\delta T_{c} / \delta \sigma_{i}\right)^{2}=\left(\Delta E / E i^{2}\right)(T c / \Delta C p)$, where $i$ is the uniaxial stress in the $i$ th direction and $T c$ is the transition temperature.

ZrTe5: Brill and Sambongi/13/ measured the change in Young's modulus in ZrTe5 as a function of temperature (Fig.14). A sharp decrease in modulus has been

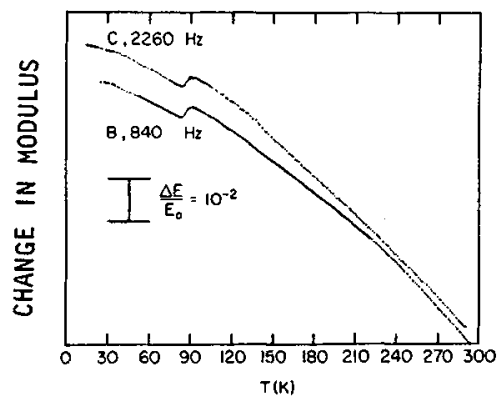

Fig.14. Relative change in Young's modulus verus temperature for $\mathrm{ZrTe}_{5} / 13 \%$

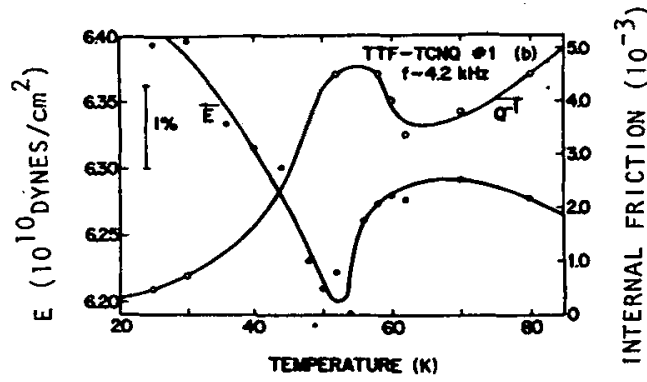

Fig.15. Internal friction for TTF.TCNQ as a function of temperature $/ 3 /$. 
observed at $84 \mathrm{~K}$. They did not observe any anomaly near $140 \mathrm{~K}$ where charge or spin density waves are formed. $\frac{111 \text { - INTERNAL FRICTION DUE TO CDW }}{\text { TRANSITIONS }}$

The internal friction exhibits a peak at CDW transitions.

ITF-TCNQ: Barmatz et al/3/ measured internal friction as a function of temperature. They found a peak near $54 \mathrm{~K}$ corresponding to the metal non-metal transition (Fig. 15). TaSe2: The internal friction in TaSe2 has been measured by Barmatz et al $16 /$. At the commensurate charge-density-wave transition near $90 \mathrm{~K}$ in $\mathrm{TaSe} 2$, the internal friction exhibit extremum, which is an order of magnitude larger than the anomalies at the incommensurate transition. At all the chargedensity-wave transitions, the internal friction maximum occurs at a lower temperature than the modulus minimum. At the transition temperature the internal friction associated with the transformation is finite and has a tail extending into the high temperature phase (Figs. 10,11, and 12). For a single relaxation process, the internal friction is represented by

$Q^{-1}[\omega, T]=A[T]\left[\omega \tau /\left(1+\omega^{2} \tau^{2}\right)\right]$

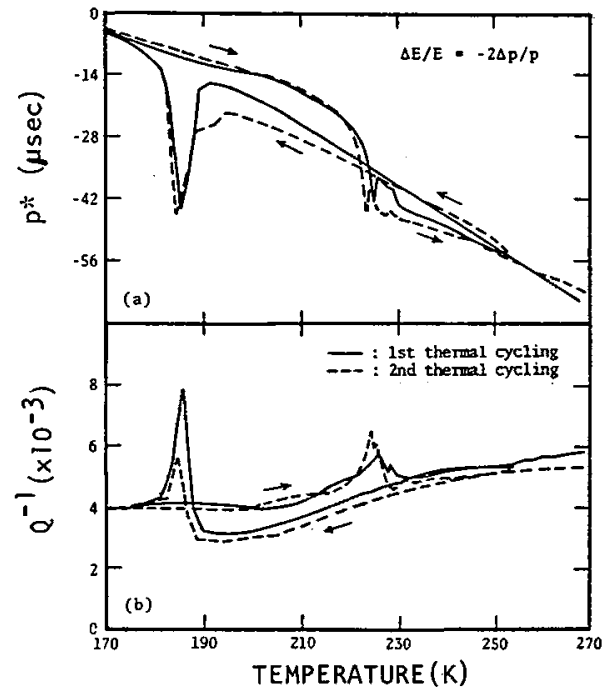

Fig.16.Internal friction and corresponding changes of the resonant period in $1 \mathrm{~T}-\mathrm{TaS}_{2} / 14 /$.

where $\tau$ is the characteristic relaxation $t$ ime for the process. The first overtone internal-friction maximum is appoximately twice as far below To as the fundamental internal friction maximum. In the loss at the transition the fundamental internal friction maximum is approximately four times larger than the first order maximum. The experimental results that for the ICDW transition in TaSe2, the acoustic loss is larger at the lower frequency. is puzzling and nontrivial. Barmatz et al $/ 6 /$ thought that such low relaxation frequencies are more characteristir of atomic loss mechanism such as diffusion point defects, dislocations, or macroscopic loss mechanism such as domain wall motion. Simpson et al $/ 7 / \mathrm{al}$ so obs i. ved at tenuation peak at $90 \mathrm{~K}$ and $12 \mathrm{IK}$.

IT-TaS2: Suzuki et al $/ 14 /$ measured the internal friction in $1 T$-TaS2 as a function of temperature. On cooling, a wide damping peak at about $188 \mathrm{~K}$ was observed, whereas on heating, a loss sharp maximum at about $220 \mathrm{~K}$ appeared in the temperature ranges corresponding to the anomalies in modulus ( $\mathrm{Fig} .16$ ). Comparing this with the heat capacity measurements, they suggested that the

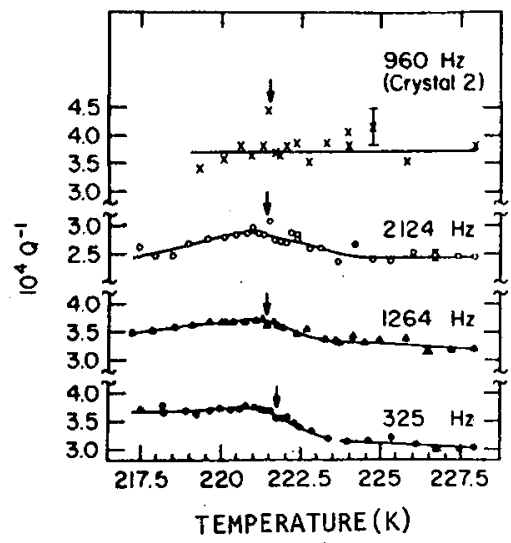

Fig.17. Internal friction as a function of temperature near the transition in $\mathrm{TaS}_{3} / 9 /$.

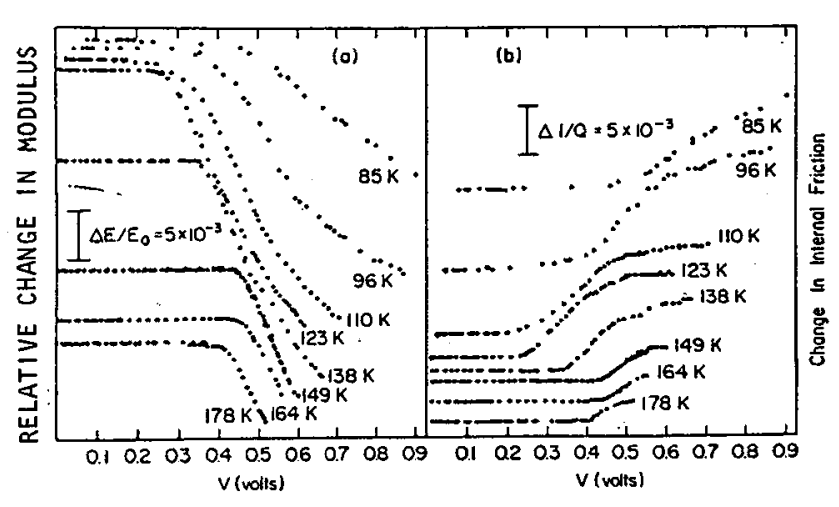

Fig.18. (a) Relative change in modulus and (b) change in $Q^{-1}$ vs voltage at several temperatures $/ 11 \%$. 

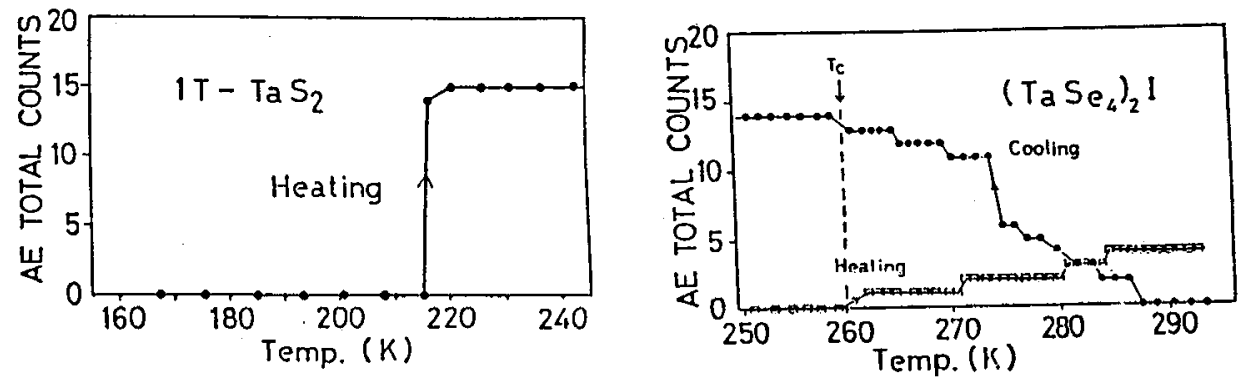

Fig.19. Acoustic emission total counts during heating in $1 \mathrm{~T}-\mathrm{T}_{\mathrm{a}} \mathrm{S}_{2}$ $/ 16 /$.

Fig. 20. Acoustic emission total counts during heating and cooling in $\left(\mathrm{TaSe}_{4}\right)_{2} \mathrm{l}$ $116 /$.

main peak in heating is preceded by a pretransition peak, while the main damping peak can be interpreted as the results of internal stress induced by the change of the crystal symmetry.

TiSe2: Attenuation peak at CDW transition has been observed $/ 15 /$.

TaS3: Brill / 9/ measured internal friction in TaS3 near the charge density wave transition (222K) (Fig. 17). Brill found that the interanl friction is sample dependent and some samples showed no anomaly at COW transition. He suggested that the internal friction in this sample is due to the presence of domain, perhaps pinned by impurities. Brill and Roark/l1/ measured the internal friction as a function of applied electric field (Fig. 18) This also indicates the CDW walls are depinned under a threshold electric field.

IV - ACOUSTIC EMISSION DURING COW TRANSITIONS

Acoustic emission may be expected when the transient elastic waves are generated in a rapid, usually localized, stress(or strain) relaxations accompanying, for example, the propagation of dislocations or the growth of cracks. Ohtake et al $/ 16 /$ performed the experiments detecting acoustic emission due to charge density waves in 1T-TaS2 and (TaSe4) 21 . Figure 19 and 20 show the total event counts of acoustic emissions in IT-TaS2 and (TaSe4)2I, respectively, as a function of temperature. The acoustic emission was detected only at the CDW transition temprature on heating in $1 \mathrm{~T}$-TaS2. In one dimensional(TaSe4) 21, acoustic emission was detected above the CDW transition temperature both cooling and heating. They suggested that the acoustic emission was caused by the motion of CDW domains by the analogy of the case of the martensitic transformation.

\section{$V$ - CDW DOMAIN WALLS}

CDW domains can be created at any lattice sites. These domains can be grown. At finite temperatures, domain size is also finite (Fig.22(a)). If this is Fourier transformed, the period has finite width. When domains in phase(Fig. $22(a))$ hit together, no domain wall is formed (Fig.22(c)), but when domains in ant $i$-phase (Fig.22(b)) hit, the CDW domain walls are formed (FIG.22(d)). This is just like anti-phase domain boundaries in the order-disorder alloys. These walls can move back and forth under alternating stress absorbing energy. This may be the reason why the internal friction shows a peak at CDW transition. Two dimensional domains have been discussed $/ 17 /$. The nucleation process has been also discussed $/ 18 /$.

VI - Summary: Change in elastic constants, internal friction peak and acoustic emission have been observed at or near the CDW transition temperature. The internal friction peak and acoustic emission near the CDW transition temperature are probably due to the motion of CDW domain walls.

ACKNOWLEDGEMENT: The author expresses his gratitude to Profs. Granato and Wert for giving him the opportunity of reporting this paper at the Conference. He also thank to Prof. R. Yamamoto, Messeurs A. Suzuki and K. Ohtake for their stimulating discussions. 
(a)

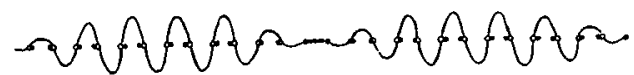

IN PHASE

(b)

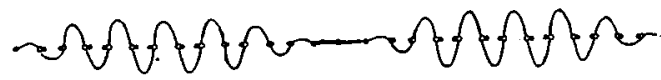

ANT I -PHASE

(c)

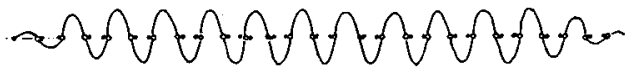

NO CDW DOMAIN

WALLS

(d)

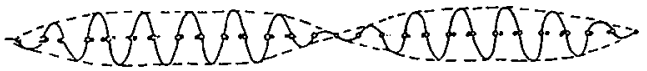

CDW ANT I -PHASE DOMAIN WALLS

Fig. 22. (a) Two COW domains in phase, (b) Two CDW domains in anti-phase (c) Two CDW domains in phase join laving no CDW domain walls, (d) Two CDW domains in anti-phase join leaving a CDW domain wall.

\section{REFERENCES}

/1/ Testardi,L.R., Phys.Rev.B12(1975) 3849.

/2/Sato, E., Ohtake, K., Yamamoto, R., Doyama, M. and Endo, K.,Proc. The 7th International Conference on Positron Annihilation, 1985.

13/ Barmatz, M., Testardi,L.R.,Garito, A. F., and Heeger, A.J., Solid State Comm $15(1974) 1299$.

14/Ishiguro,T., Kagoshima, S. and Anzai, H.,J. Soc. Japan 42(1977) 365.

15/ Tiedje,T., Haering, R. R., Jericho, M. H., Roger,W. A. and Simpson, A., Solid State Comm 23 (1977) 713.

16/ Barmatz,M.,Testardi, L. R., and Di Salvo,F.J., Phys. Rev. B 12 (1975) 4367.

$17 /$ Simpson, A.M., Jericho,M.H.,DiSalvo,F.J., Solid State Comm.44(1982) 1543.

18/ Skolnick, M.S., Roth, S.and Alms,H.,J.Phys.C 10(1977) 2523.

19/ Brill,J.W., Solid State Comm. 41 (1982) 925.

110/Zetti,A.,Gruner and Thompson, A. H.,Phys. Rev.B 26 (1982) 5760.

/11/ Brill, J.W. and Roark,W., Phys. Rev.Lett. 53(1984) 846.

$112 /$ Brill,J.W.and Ong,N.P., Solid State Comm.25 (1978) 1075.

/13/ Brill,J.W. and Sambongi,T.,J.Phys. Soc.Japan 53(1984) 20.

$114 /$ Suzuki, A., Yamamoto, R., Doyama, M. ,Mizubayashi,H., and Okuda, S. ,This Conference.

115/ Caille,A., Lepine,Y., Tericho, M.H., and Simpson, A.M., Phys.Rev. B28(1983) 5454.

116/ Ohtake,K., Sato, E., Suzuki, Y., Yamamoto, R., Doyama, M., Endo, K., Wakayama, S., and Kishi,T., This Conference.

/17/ Nakanishi,K., and Shiba,H., J. Phys. Soc. Japan 53 (1984) 1103.

118/ Prelowzek, P. and Rice, T. M., J. Phys. C 16 (1983) 6513. 